\title{
On the Deflection of Asteroids with Mirrors
}

\author{
Massimiliano Vasile • Christie Alisa Maddock
}

Received: date / Accepted: date / Draft: April 20, 2010

\begin{abstract}
This paper presents an analysis of an asteroid deflection method based on multiple solar concentrators. A model of the deflection through the sublimation of the surface material of an asteroid is presented, with simulation results showing the achievable orbital deflection with, and without, accounting for the effects of mirror contamination due to the ejected debris plume. A second model with simulation results is presented analyzing an enhancement of the Yarkovsky effect, which provides a significant deflection even when the surface temperature is not high enough to sublimate. Finally the dynamical model of solar concentrators in the proximity of an irregular celestial body are discussed, together with a Lyapunov-based controller to maintain the spacecraft concentrators at a required distance from the asteroid.
\end{abstract}

Keywords Asteroid Deflection · Formation Flying · Orbit Control · Yarkovsky Effect

PACS 96.30.Ys $\cdot 45.50 . \mathrm{Pk}$

\section{Introduction}

Over the last few years, the possible scenario of an asteroid threatening to impact the Earth has stimulated intense debate among the scientific community about possible deviation methods. Small celestial bodies like Near Earth Objects (NEO) have become a common subject of study because of their importance in uncovering the mysteries

This research is partially supported by the ESA/ESTEC Ariadna study 08/4301, contract number: 21665/08/NL/CB [40].

M. Vasile

Space Advanced Research Team Department of Aerospace Engineering, University of Glasgow Tel.: +44-141-3306465

Fax: +44-141-3305560

E-mail: m.vasile@aero.gla.ac.uk

C. Maddock

Space Advanced Research Team Department of Aerospace Engineering, University of Glasgow Tel.: +44-141-3308470

Fax: +44-141-3305560

E-mail: c.maddock@aero.gla.ac.uk 
of the formation, evolution and composition of the solar system. Among all asteroids, NEO's have stepped into prominence because of two important aspects: they are among the easiest celestial bodies to reach from Earth, in some cases, they can be reached with less demanding trajectories than a simple Earth-Moon trajectory and, even more meaningful, they may represent a threat to our planet.

As of January 2010, there are 1087 known Potentially Hazardous Asteroids (PHA) out of a current total of 8396 detected NEOs. Of the PHAs detected, 145 are estimated to be over $1 \mathrm{~km}$ in diameter [15]. For NEO's between 50 and $100 \mathrm{~m}$ in diameter (the lower limit of an object's atmospheric penetration), it is estimated there are over half a million NEO's with an impact frequency of one per thousand years [26]. Each of these impacts permanently alters the characteristics of our planet to varying degrees. These events, and the risks they pose to our fragile ecosystem, have made the space community turn their attention to the issue of NEO's. Evidence of this new found interest is the prolific and successful asteroid exploration program of the last decade, with many completed missions such as NEAR [21], Deep Space 1 [29], Deep Impact [13] and Stardust [38], ongoing missions like Rosetta [11], Hayabusa [24] and Dawn [32] and future missions such as Marco Polo [1]

In order to predict the effects of a deflection strategy, some studies have addressed the asteroid deviation problem either with an analytical approach $[7,39,16,35,8]$ or by means of a numerical procedure based on a $n$-body model [4]. Other studies addressed the optimal design of transfer trajectories to asteroids $[28,6]$.

A few authors have performed a partial comparative assessment of the numerous proposed mitigation strategies [12]. In a recent work by Sanchez et al. [34] a comprehensive set of deflection methods were compared according to the following criteria: mass into space, achievable deflection, warning time and technology readiness. No ideal scenario was considered but a large number of realistic mission options, including launch and transfer to the asteroid, were simulated.

¿From the comparison, the conclusion was that nuclear stand-off explosions were the most effective on the widest range of asteroids. The second best was solar sublimation with all the other methods order of magnitude less effective (according to the proposed comparison criteria). Although nuclear explosions were the most effective, a subsequent study by Sanchez et al. [33] demonstrated that for both nuclear explosions and kinetic impacts, a resulting fragmentation of the asteroid could potentially increase the risk of impact(s) on the Earth.

This paper presents an analysis of the performance of a solar ablation-based technique for the deflection of NEO's. The paper starts with an introduction to the concept of solar sublimation, followed by a deflection model. The third section presents a model of the achievable deflection due to a combination of the Yarkovsky effect [41] and solar radiation pressure on the mirror surfaces. A fourth section follows with some results on the achievable deviation with and without considering the contamination of the solar concentrators and with the combined Yarkovsky and solar pressure effects. The paper concludes with the derivation of a Lyapunov controller to maintain the solar concentrators in the proximity of the asteroid, as dictated by the orbital dynamic model.

\section{The solar sublimation concept}

In 1992 Lunan et al. [19] and more extensively in 1993, Melosh et al. [22, 23] proposed the use of a mirror (solar concentrator) to focus the solar energy onto a small portion 
of the surface of an asteroid. The resulting heat would sublimate the surface material creating a jet of gas and dust that would produce a continuous thrust. A conceptually similar idea is to use a laser beam, either powered by a nuclear reactor or solar arrays, to induce the required sublimation of the surface material $[27,43]$.

In a more recent study, Kahle et al. [17] pointed out a number of technological limitations and considerations on the basic solar collector idea proposed by Melosh et al. In particular:

- If the light of the Sun is focused directly onto the surface of the asteroid, in order to have a high enough power density the mirror should be at relatively close distance from the asteroid, e.g. a separation distance of $1.25 \mathrm{~km}$ for a $630 \mathrm{~m}$ diameter mirror. As a consequence, the mirror should operate and manoeuvre under the effect of the irregular gravity field of the asteroid. Furthermore, at such a distance the contamination of the primary mirror, due to the ejected gasses, would be significant. A longer distance would imply a larger mirror with a consequent increased difficulty in the control of the attitude.

- If a secondary steering mirror is used, the contamination of the primary can be reduced but the secondary would suffer the full contamination problem. Kahle et al. proposed some solutions to the contamination issue but all imply a significant increase in the complexity and mass of the system.

- The deployment and control of a large mirror represents a significant technological challenge and, moreover, a single point failure for the entire mission.

- The total light pressure on the primary mirror would induce a significant force on the spacecraft requiring constant orbit control.

- The high level of solar power collected by the primary reflector would force the secondary reflector to operate at extremely high temperatures, in particular if the surface is contaminated. When this happens, absorptivity is increased, causing a further reduction in reflectivity.

The deflection model presented in this paper includes the effect of the contamination of the solar concentrators according to the contamination model proposed by Kahle et al. [17]. Rather than considering a single large mirror, we will analyze the case of multiple smaller mirrors, each superimposing their beams on the same small spot on the surface of the asteroid.

\subsection{Deflection Model}

The asteroid 99942 Apophis is chosen as a test case based on its degree of threat. Compared to other near Earth objects, Apophis has a relatively high probability of impacting the Earth in April 2036, although the actual cumulative impact probability is low, only $2.2 \times 10^{-5}$ [10]. Whether the asteroid will impact the Earth is contingent upon the asteroid's fly-by of Earth in 2029. During that event Apophis could pass through a gravitational keyhole, a precise region in space no more than about $400 \mathrm{~m}$ across, which would set up resonances that would increase the probability of future impacts starting on 13 April 2036.

As with nearly all NEO's, the orbital data for Apophis has been gained from Earthbased observations, which are limited based on the visibility of the asteroid from the astronomy station, availability of the station, etc. [5]. As a result, the present knowledge of the orbit of Apophis is not good enough to provide an accurate long term prediction 


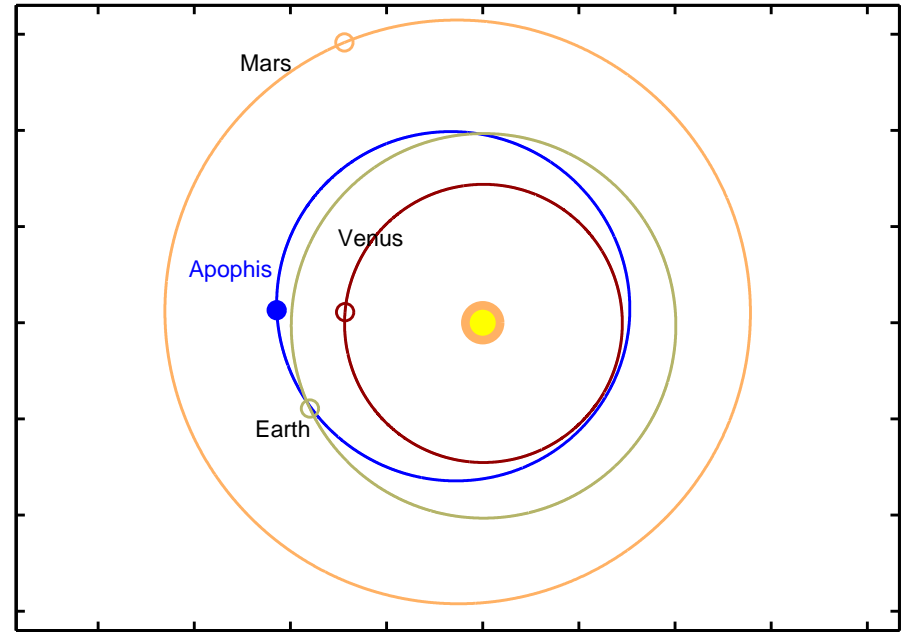

(a) View of the ecliptic plane

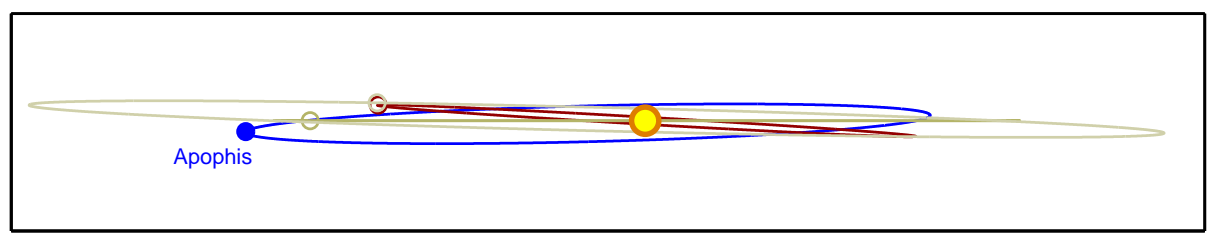

(b) Inclination difference between the orbits

Fig. 1: Orbit of the NEO Apophis, compared to the orbits of the Mars, Earth and Venus.

of its evolution. This underlines the need for longer term measurements from a spacebased platform [36].

Table 1 give the orbital and physical data of the asteroid used in this study, and if known, their estimated uncertainty [25]. The dimensions for the asteroid shape are estimated using an ellipsoidal model,

$$
a_{\mathrm{I}}=\sqrt{2} d_{\mathrm{A}} \quad b_{\mathrm{I}}=d_{\mathrm{A}} \quad c_{\mathrm{I}}=\frac{d_{\mathrm{A}}}{\sqrt{2}}
$$

where $a_{\mathrm{I}} \geq b_{\mathrm{I}} \geq c_{\mathrm{I}}$ are the three radii along the three orthogonal axes [9], and $d_{\mathrm{A}}$ is estimated average diameter based on the observed magnitude, given in Table 1. Fig. 1 shows the orbit of Apophis relative to nearby planets.

The minimum orbital intersection distance (MOID) is the separation distance at the closest point between two orbits, e.g. Apophis and the Earth. The deviation distance is defined here as the difference in position between the original, undeviated orbit $\mathbf{k}_{\mathrm{A}_{0}}$ and the deviated orbit $\mathbf{k}_{\mathrm{A}_{d e v}}$ at $t_{\mathrm{MOID}}$ [7] (see Fig. 2). Fig. 3 illustrates the definition and nomenclature for the references frames used here.

Non-linear equations for were derived for determining the deviation vector $\Delta \mathbf{r}_{d e v}=$ $\mathbf{r}_{\mathrm{A}_{\text {dev }}}-\mathbf{r}_{\mathrm{A}_{0}}$ as a function of the ephemeris in the Hill reference frame $\mathcal{A}$ centred on 
Table 1: Estimated and observed orbital and physical properties of Apophis 99942 [25].

\begin{tabular}{llll}
\hline \multicolumn{1}{c}{ Element } & & Measured Value & Uncertainty, $\sigma$ \\
\hline Semi-major axis & $a$ & $0.922438 \mathrm{AU}$ & $2.3613 \times 10^{-8}$ \\
Eccentricity & $e$ & 0.191204 & $7.6074 \times 10^{-8}$ \\
Inclination & $i$ & $3.331420 \mathrm{deg}$ & $2.0238 \times 10^{-6}$ \\
Right ascension of the ascending node & $\Omega$ & $204.442505 \mathrm{deg}$ & 0.00010721 \\
Argument of periapsis & $\omega$ & $126.404227 \mathrm{deg}$ & 0.00010632 \\
Period & $T_{\mathrm{A}}$ & $323.596917 \mathrm{~d}$ & $1.2426 \times 10^{-5}$ \\
Mean motion & $n_{\mathrm{A}}$ & $1.112495 \mathrm{deg} / \mathrm{d}$ & $4.2718 \times 10^{-8}$ \\
\hline Diameter & $d_{\mathrm{A}}$ & $270 \mathrm{~m}$ & $60 \mathrm{~m}$ \\
Mass & $m_{\mathrm{A}}$ & $2.7 \times 10^{10} \mathrm{~kg}$ & \\
Gravitational constant & $\mu_{\mathrm{A}}$ & $1.801599 \times 10^{-9} \mathrm{~km}^{3} / \mathrm{s}^{2}$ & \\
Rotational velocity & $w_{\mathrm{A}}$ & $5.8177 \times 10^{-5} \mathrm{rad} / \mathrm{s}$ & \\
\hline
\end{tabular}

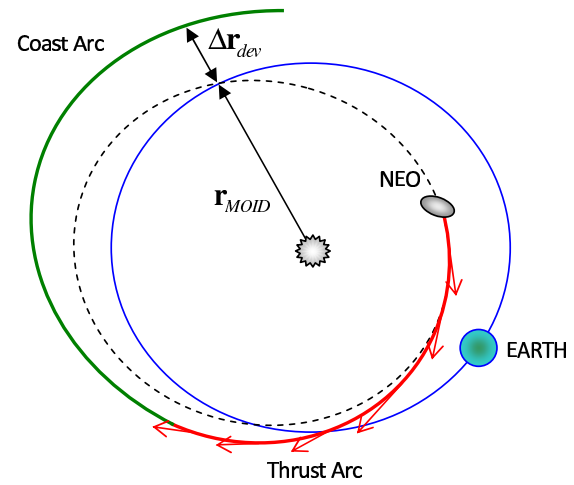

Fig. 2: Definition of deviation distance at the MOID.

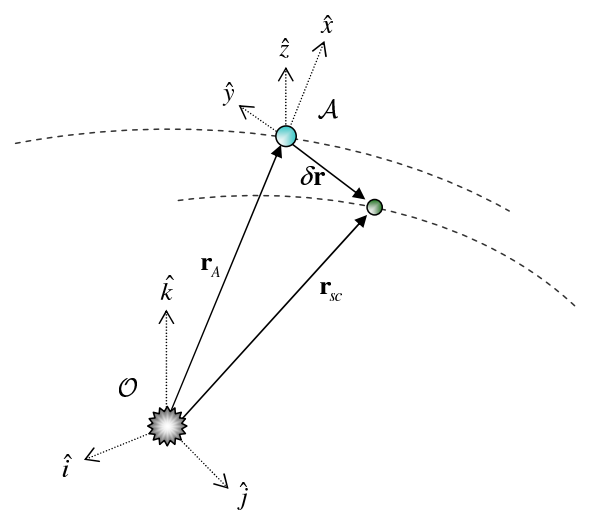

Fig. 3: Definition of the reference frames, including the rotating, relative Hill frame $\mathcal{A}$ centered on the asteroid.

the asteroid [20], with $\Delta \mathbf{k}=\mathbf{k}_{\mathrm{A}_{d e v}}-\mathbf{k}_{\mathrm{A}_{0}}=[\Delta a, \Delta e, \Delta i, \Delta \Omega, \Delta \omega, \Delta M]^{\mathrm{T}}$ giving the difference in Keplerian parameters between the undeviated and deviated orbit.

$$
\begin{gathered}
\Delta \mathbf{r}_{d e v}=r_{\mathrm{A}_{d e v}} \Psi-\left[\begin{array}{c}
r_{\mathrm{A}_{0}} \\
0 \\
0
\end{array}\right] \\
\Psi=\left[\begin{array}{c}
\xi \cos \left(\theta_{\mathrm{A}_{0}}+\Delta \theta\right)+\sin \left(\theta_{\mathrm{A}_{0}}+\Delta \theta\right)\left(\varrho \sin \theta_{\mathrm{A}_{0}}-\cos \left(i_{\mathrm{A}_{0}}+\Delta i\right) \sin \Delta \Omega \cos \theta_{\mathrm{A}_{0}}\right) \\
-\zeta \cos \left(\theta_{\mathrm{A}_{0}}+\Delta \theta\right)+\sin \left(\theta_{\mathrm{A}_{0}}+\Delta \theta\right)\left(\varrho \cos \theta_{\mathrm{A}_{0}}+\cos \left(i_{\mathrm{A}_{0}}+\Delta i\right) \sin \Delta \Omega \sin \theta_{\mathrm{A}_{0}}\right) \\
-\cos \left(\theta_{\mathrm{A}_{0}}+\Delta \theta\right) \sin \Delta \Omega \sin i_{\mathrm{A}_{0}}+\varpi \sin \left(\theta_{\mathrm{A}_{0}}+\Delta \theta\right)
\end{array}\right]
\end{gathered}
$$

where $\Psi$ is the coordinate system transformation matrix, $\mathbf{k}=[a, e, i, \Omega, \omega, \nu]^{\mathrm{T}}$ are the set of standard Keplerian elements with the true anomaly $\nu, \theta=\nu+\omega$ is the true 
latitude, and,

$$
\begin{aligned}
\varpi & =\cos i_{\mathrm{A}_{0}} \sin \left(i_{\mathrm{A}_{0}}+\Delta i\right)-\cos \Delta \Omega \cos \left(i_{\mathrm{A}_{0}}+\Delta i\right) \sin i_{\mathrm{A}_{0}} \\
\varrho & =\sin i_{\mathrm{A}_{0}} \sin \left(i_{\mathrm{A}_{0}}+\Delta i\right)+\cos \Delta \Omega \cos \left(i_{\mathrm{A}_{0}}+\Delta i\right) \cos i_{\mathrm{A}_{0}} \\
\xi & =\cos \Delta \Omega \cos \theta_{\mathrm{A}_{0}}+\cos i_{\mathrm{A}_{0}} \sin \Delta \Omega \sin \theta_{\mathrm{A}_{0}} \\
\zeta & =\cos \Delta \Omega \sin \theta_{\mathrm{A}_{0}}-\cos i_{\mathrm{A}_{0}} \sin \Delta \Omega \cos \theta_{\mathrm{A}_{0}}
\end{aligned}
$$

The change in the orbital parameters is calculated by numerically integrating the Gauss planetary equations using a thrust vector $\mathbf{u}_{d e v}=\left[\begin{array}{lll}u_{v} & u_{n} & u_{h}\end{array}\right]^{\mathrm{T}}$ induced by the deflection method:

$$
\Delta k=\int_{t_{0}}^{t_{i}} \frac{d \mathbf{k}\left(\mathbf{u}_{d e v}\right)}{d t} d t
$$

In the following we will assume that the deflection action is always aligned with the heliocentric velocity of the asteroid, therefore $u_{n}=0$ and $u_{h}=0$. The Gauss equations k are given by [2],

$$
\begin{aligned}
\frac{d a}{d t} & =\frac{2 a^{2} v}{\mu_{\mathrm{A}}} u_{v} \\
\frac{d e}{d t} & =\frac{1}{v}\left(2(e+\cos \nu) u_{v}-\frac{r_{\mathrm{A}} \sin \nu}{a} u_{n}\right) \\
\frac{d i}{d t} & =\frac{r_{\mathrm{A}} \cos \theta}{h} u_{h} \\
\frac{d \Omega}{d t} & =\frac{r_{\mathrm{A}} \sin \theta}{h \sin i} u_{h} \\
\frac{d \omega}{d t} & =\frac{1}{e v}\left(2 \sin \nu u_{v}+\left(2 e+\frac{r_{\mathrm{A}}}{a \cos \nu}\right) u_{n}\right)-\frac{r_{\mathrm{A}} \sin \theta \cos i}{h \sin i} u_{h} \\
\frac{d \nu}{d t} & =\frac{h}{r^{2}}-\frac{1}{e v}\left(2 \sin \nu u_{v}+\left(2 e+\frac{r_{\mathrm{A}}}{a} \cos \nu\right) u_{n}\right) \\
\frac{d M}{d t} & =n_{\mathrm{A}}-\frac{\sqrt{a p}}{e a v}\left(2 \sin \nu\left(1+e^{2} \frac{r_{\mathrm{A}}}{p}\right) u_{t}+\frac{r_{\mathrm{A}} \cos \nu}{a} u_{n}\right)
\end{aligned}
$$

with,

$$
p=a \sqrt{1+e} \quad v=\sqrt{\frac{2 \mu_{\odot}}{r_{\mathrm{A}}}-\frac{\mu_{\odot}}{a}} \quad n_{\mathrm{A}}=\sqrt{\frac{\mu_{\odot}}{a^{3}}}
$$

where $n_{\mathrm{A}}$ is the mean orbital motion of the asteroid, and $\mu_{\odot}$ is the gravitational constant of the Sun.

Colombo et al. [7] determined that the change in angular location, in this case given by the mean anomaly, calculated at the MOID is,

$$
\Delta M=\int_{t_{0}}^{t_{i}} \frac{d M}{d t} d t+n_{\mathrm{A}_{0}}\left(t_{0}-t_{\mathrm{MOID}}\right)+n_{\mathrm{A}_{i}}\left(t_{\mathrm{MOID}}-t_{i}\right)
$$

Eq. (2) together with Eqs. (5) and (6) give the deviation of the orbit of the asteroid at the time of the MOID, regardless of the actual position of the Earth, but not the close approach or the minimum orbit interception distance with respect to the Earth. Vasile and Colombo [39] demonstrated that a good estimation of the minimum interception distance can be obtained by projecting the deviation onto the $b$-plane of the Earth at the time of the MOID (assuming that the Earth is at the point of the MOID). In 
the test section, therefore, we will present the variation of the impact parameter (or $b$ parameter) on the $b$-plane of the Earth.

The thrust produced by the deflection method is a direct function of the rate of the expelled surface matter [34],

$$
\frac{d m_{\text {exp }}}{d t}=2 n_{s c} v_{\text {rot }} \int_{y_{0}}^{y_{\max }} \int_{t_{\text {in }}}^{t_{\text {out }}} \frac{1}{H}\left(P_{\text {in }}-Q_{\text {rad }}-Q_{\text {cond }}\right) d t d y
$$

where $\left[t_{\text {in }}, t_{\text {out }}\right]$ is the duration for which a point is illuminated, $\left[y_{0}, y_{\max }\right]$ are the limits of the vertical illuminated surface area (i.e. orthogonal to the direction of rotation of the asteroid), $H$ is the enthalpy of sublimation, $v_{\text {rot }}$ is the linear velocity of a point as it travels horizontally (i.e., orthogonal to $y$ ) through the illuminated spot area and $n_{s c}$ is the number of spacecraft in the formation.

The input power per unit area due to the solar concentrators is given by,

$$
P_{i n}=\sigma_{s y s} C_{r}\left(1-\varsigma_{\mathrm{A}}\right) S_{0}\left(\frac{r_{\mathrm{AU}}}{r_{\mathrm{A}}}\right)^{2}
$$

where $\varsigma_{\mathrm{A}}=0.2$ is the albedo of the asteroid, $S_{0}=1367 \mathrm{~W} / \mathrm{m}^{2}$ is the solar flux at $1 \mathrm{AU}$ and scaled to the Sun-asteroid distance $r_{\mathrm{A}}, \sigma_{s y s}$ is the system efficiency, and $C_{r}$ is the concentration ratio (the ratio between the power density from the Sun on the mirror surface, and that of the spot area on the asteroid).

The heat loss due to black-body radiation and the conduction loss are defined, respectively, as,

$$
\begin{aligned}
Q_{\text {rad }} & =\sigma \varepsilon_{b b} T^{4} \\
Q_{\text {cond }} & =\left(T_{\text {subl }}-T_{0}\right) \sqrt{\frac{c_{\mathrm{A}} k_{\mathrm{A}} \rho_{\mathrm{A}}}{\pi t}}
\end{aligned}
$$

where $\sigma$ is the Stefan-Boltzmann constant, $\epsilon_{b b}$ is the black body emissivity, $T$ is the temperature and $c_{\mathrm{A}}, \rho_{\mathrm{A}}$ and $k_{\mathrm{A}}$ are, respectively, the heat capacity, density and thermal conductivity of the asteroid. For the asteroid Apophis, $c_{\mathrm{A}}=750 \mathrm{~J} / \mathrm{kg} \cdot \mathrm{K}$ based on the average value for silicate materials, $k_{\mathrm{A}}=2 \mathrm{~W} / \mathrm{K} / \mathrm{m}$ and $\rho_{\mathrm{A}}=2600 \mathrm{~kg} / \mathrm{m}^{3}$ [30]. The sublimation temperature assumed is that for forsterites, $T_{\text {subl }}=1800 \mathrm{~K}$ [42], with $T_{0}$ set to $278 \mathrm{~K}$. The induced acceleration due to the sublimation process can then determined by [34],

$$
\mathbf{u}_{s u b}=\frac{\Lambda \bar{v} \dot{m}_{\exp }}{m_{\mathrm{A}}} \hat{\mathbf{v}}_{\mathrm{A}}
$$

where $\hat{\mathbf{v}}_{\mathrm{A}}$ is direction of velocity vector of the NEO, $\Lambda \simeq\left(\frac{2}{\pi}\right)$ is the scattering factor, $\bar{v}$ is the average velocity of the debris particles according to Maxwell's distribution of an ideal gas:

$$
\bar{v}=\sqrt{\frac{8 \mathrm{k}_{\mathrm{B}} T_{\text {subl }}}{\pi \mathrm{M}_{\mathrm{Mg} g_{2} \mathrm{SiO}_{4}}}}
$$

where $\mathrm{k}_{\mathrm{B}}$ is the Boltzmann constant, and $\mathrm{M}_{\mathrm{Mg}_{2} \mathrm{SiO}_{4}}$ is the molecular mass of fosterite. The scattering factor is computed as the average of all possible thrust directions assuming that the thrust can point randomly at any angle $\alpha_{\mathrm{T}}$ between 0 and $\pi$, therefore $\Lambda=\frac{1}{\pi} \int_{0}^{\pi} \cos \alpha_{\mathrm{T}} d \alpha_{\mathrm{T}}$ [34]. Note that some preliminary experiments demonstrate that the plume is actually more focused. However, assuming a uniform distribution of the thrust pointing direction over an angle of 180 degrees is a conservative choice.

The remaining mass of the asteroid $m_{\mathrm{A}}$ is calculated by numerically integrating Eq. (7). This induced acceleration $\mathbf{u}_{\text {dev }}$ is used with the Gauss equations in Eq. (5) in order to determine the change in the NEO orbit due to the solar sublimation. 


\subsection{Contamination Model}

The contamination of the mirror surfaces due to the debris plume is modeled based on the work by Kahle et al. [17] The study is based on a number of initial assumptions regarding the expansion of the plume and sublimation process. The first assumption holds that the sublimation process is comparable to the generation of tails in comets. The asteroid is assumed to contain a reservoir of material underneath the surface, with the gas expanding both outwards as expected, and inwards through a throat into vacuum within the asteroid itself. This assumption holds true, for example, for a loose rubble-pile asteroid model. The second assumption is that the plume expansion is similar to the expansion of gas of a rocket engine outside the nozzle.

The density of the expelled gas $\rho_{\text {exp }}$ is computed analytically,

$$
\rho_{\text {exp }}\left(r_{s / s c}, \varphi\right)=j_{c} \frac{\dot{m}_{\text {exp }}}{\bar{v} A_{\text {spot }}}\left(\frac{d_{\text {spot }}}{2 \delta r+d_{\text {spot }}}\right)^{2}(\cos \Theta)^{2 /(\kappa-1)}
$$

where $r_{s / s c}$ is the distance from the spot on the surface of the asteroid to the spacecraft, and $\Theta=\pi \varphi / 2 \varphi_{\max }$ where $\varphi$ is the angle between the spot-spacecraft vector and the $y$ axis of the Hill reference frame. The jet constant $j_{c}$ was set to 0.345 , the maximum expansion angle $\varphi_{\max }=130.45^{\circ}$, and adiabatic index $\kappa=1.4$, based on the values for diatomic particles [18].

The position vector $\mathbf{r}_{s / s c}$ from the spot to the spacecraft is defined as:

$$
\mathbf{r}_{s / s c}=\left[\begin{array}{c}
x+r_{e l l} \sin w_{\mathrm{A}} t \cos \left(w_{\mathrm{A}} t+\theta_{v_{\mathrm{A}}}\right)-r_{e l l} \cos w_{\mathrm{A}} t \sin \left(w_{\mathrm{A}} t+\theta_{v_{\mathrm{A}}}\right) \\
y-r_{e l l} \cos w_{\mathrm{A}} t \cos \left(w_{\mathrm{A}} t+\theta_{v_{\mathrm{A}}}\right)-r_{e l l} \sin w_{\mathrm{A}} t \sin \left(w_{\mathrm{A}} t+\theta_{v_{\mathrm{A}}}\right) \\
z
\end{array}\right]
$$

where the radius of the ellipse is given by,

$$
r_{e l l}=\frac{a_{\mathrm{I}} b_{\mathrm{I}}}{\sqrt{\left(b_{\mathrm{I}} \cos \left(w_{\mathrm{A}} t+\theta_{v_{\mathrm{A}}}\right)\right)^{2}+\left(a_{\mathrm{I}} \sin \left(w_{\mathrm{A}} t+\theta_{v_{\mathrm{A}}}\right)\right)^{2}}}
$$

and, with reference to Fig. 3, the position of the spacecraft with respect to the center of the asteroid is $\delta \mathbf{r}=[x, y, z]^{T}$. We assume here that the asteroid is spinning around the $z$ axis with a rotational velocity $w_{\mathrm{A}}$. The direction of the velocity of the asteroid in the heliocentric reference frame projected onto the Hill reference frame $\mathcal{A}$ is $\theta v_{\mathrm{A}}$. In other words, in order to have a deflection thrust aligned with the velocity of the asteroid, the spot is assumed to be at an elevation angle over the $y$ axis equal to $\theta v_{\mathrm{A}}$.

The third assumption made is that all the particles impacting the surface of the mirror condense and stick to the mirror. The exhaust velocity is constant, therefore the thrust depends only on the mass flow. A higher thrust results in a higher mass flow and thus in a faster contamination. Following the approach used to compute the contamination of surfaces due to out-gassing, a view factor $\psi_{v f}$ was added equal to the angle between the normal to the mirror and the incident flow of gas. The resulting variation of the thickness of the material condensing on the mirror can be computed with:

$$
\frac{d h_{c n d}}{d t}=\frac{2 \bar{v} \rho_{\text {exp }}}{\rho_{\text {layer }}} \cos \psi_{v f}
$$

The average debris velocity $\bar{v}$ is multiplied by a factor of 2 to account for the expansion of the gas in a vacuum. The layer density $\rho_{\text {layer }}$ is to set to $1 \mathrm{~g} / \mathrm{cm}^{3}$. The power 
density on the asteroid surface is decreased based on the contamination of the mirrors. A degradation factor $\tau$ is applied to the power beamed to the asteroid surface, based on the Lambert-Beer-Bouguer law [17],

$$
\tau=\exp ^{-2 \eta h_{c n d}}
$$

where $\eta=10^{4} / \mathrm{cm}$ is the absorption coefficient for forsterite. Eq. (16) is numerically integrated, along with the Gauss equations in (5), for the period of the mission.

\subsection{Tugging Effect}

The spacecraft will librate at a distance $\delta r$ from the asteroid, thus exerting a tugging effect on it [37]. The tugging acceleration $\mathbf{u}_{t u g}$ is given by:

$$
\mathbf{u}_{t u g}=-n_{s c} \frac{G m_{s c}}{\delta r^{2}} \delta \hat{\mathbf{r}}
$$

where $G$ is the universal gravity constant. The mass of the spacecraft $m_{s c}$ is dictated mainly by the overall mass of the mirror. It is assumed here that the mirror has an area density of $0.6 \mathrm{~kg} / \mathrm{m}^{2}$, and the dry mass of the bus is $500 \mathrm{~kg}$ [40]. A $30 \%$ margin is added both to the total mass of the mirror and the overall mass of the spacecraft (i.e., mirror plus bus) for contingency and orbit maintenance. The distance from the asteroid depends on the required concentration ratio $C_{r}$. If the distance is constant the concentration ratio will change with the distance from the Sun. On the contrary, if the concentration ratio is kept constant then the spacecraft will have to move back and forth towards the asteroid according to:

$$
\delta \mathbf{r}=\frac{d_{s p o t}}{2 \vartheta_{\odot}} \frac{r_{s c}}{r_{\mathrm{AU}}}\left[\begin{array}{c}
\cos \epsilon_{\mathrm{AEP}} \\
\sin \epsilon_{\mathrm{AEP}} \\
0
\end{array}\right]
$$

where $d_{\text {spot }}$ is the desired diameter of the illuminated spot area and $\vartheta_{\odot}$ is the angular radius of the Sun (at one AU, $\vartheta_{\odot}=4.53 \mathrm{mrad}$ ). The angle $\epsilon_{\mathrm{AEP}}$ is the elevation over the $y$ axis. Note that the movement should be librating inline the spot vector, but in this paper we adopt the simplified movement in Eq. 19.

As an example, if we assume a concentration ratio $C_{r}=100$ at aphelion with a $62 \mathrm{~m}$ diameter mirror, we need to place the mirrors $750 \mathrm{~m}$ from the surface of the asteroid which translates into a minimum distance of $890 \mathrm{~m}$ from its center (considering $\left.b_{\mathrm{I}}\right)$. Thus, the tug acceleration at aphelion is $u_{t u g, a}=2.52 \times 10^{-13} \mathrm{~m} / \mathrm{s}^{2}$ and at perihelion is $u_{t u g, p}=4.83 \times 10^{-13} \mathrm{~m} / \mathrm{s}^{2}$. If the concentration ratio is reduced to $C_{r}=35$ the minimum distance at perihelion can be brought up to about $1000 \mathrm{~m}$ and therefore the tug acceleration becomes $u_{t u g, p}=2.0 \times 10^{-13} \mathrm{~m} / \mathrm{s}^{2}$ at perihelion and $u_{\text {tug }, p}=1.24 \times 10^{-13} \mathrm{~m} / \mathrm{s}^{2}$ at aphelion.

\section{Enhanced Yarkovsky Effect}

If the power density is not high enough to sublimate the surface material, it may be sufficient to heat up the surface creating a low thrust by means of the Yarkovsky effect. 
Given the emissivity $\epsilon_{\mathrm{A}}$ of the asteroid, the force due to the light projected onto the asteroid is,

$$
\begin{aligned}
F_{\text {light }} & =2\left(1-\varsigma_{\mathrm{A}}\right) n_{s c} \Lambda_{\text {light }} \sigma_{\text {sys }} C_{r} A_{\text {spot }} S_{\text {srp }}\left(\frac{r_{\mathrm{AU}}}{r_{s c}}\right)^{2}+n_{s c} \varsigma_{\mathrm{A}} \sigma_{s y s} C_{r} A_{\text {spot }} S_{\text {srp }}\left(\frac{r_{\mathrm{AU}}}{r_{s c}}\right)^{2} \\
& =n_{s c} \sigma_{s y s} A_{\mathrm{M}} S_{s r p}\left(\frac{r_{\mathrm{AU}}}{r_{s c}}\right)^{2}\left(2 \Lambda_{\text {light }}\left(1-\varsigma_{\mathrm{A}}\right)+\varsigma_{\mathrm{A}}\right)
\end{aligned}
$$

where $S_{\text {srp }}=4.562 \times 10^{-6} \mathrm{~N} / \mathrm{m}$ is the solar pressure at one astronomical unit, $A_{\text {spot }}$ is the $2 \mathrm{D}$ illuminated surface area on the asteroid, $A_{\mathrm{M}}$ is the effective surface area of the primary mirror that is perpendicular to the Sun, $C_{r}=A_{\mathrm{M}} / A_{\text {spot }}$ is the concentration ratio, $\Lambda_{\text {light }}=4 /(3 \pi)$ is the scattering factor and $n_{\text {spot }}$ is the number of illuminated spot areas. The first component is the reflected light and the second component is the absorbed light. The scattering factor is the average of the reflected force assuming that the reflection can have any direction angle $\alpha_{\mathrm{L}}$ between $[0, \pi]$. Therefore, the scattering factor here is defined as $\Lambda_{\text {light }}=\frac{1}{\pi} \int_{0}^{\pi} \cos ^{3} \alpha_{\mathrm{L}} d \alpha_{\mathrm{L}}$. The number of spots $n_{\text {spot }}$ is equal to the number of spacecraft if no sublimation is attempted, and equal to 1 if the spacecraft tries to induce a sublimation of the surface.

For an asteroid surface temperature $T_{\mathrm{A}}$, the emission of photons will add a force component $F_{\text {ir }}[3]$,

$$
F_{\text {ir }}=n_{\text {spot }} \Lambda \frac{\epsilon_{\mathrm{A}} \sigma T_{\mathrm{A}}^{4}}{c} A_{\text {spot }}
$$

where $c$ is the speed of light. The temperature of the spot surface can be computed with the simple one-dimensional model [34]:

$$
\chi_{\mathrm{A}} \frac{\partial^{2} T}{\partial x^{2}}=\frac{\partial T}{\partial t}
$$

where $\chi_{\mathrm{A}}$ is the thermal diffusivity of the material given by,

$$
\chi_{\mathrm{A}}=\frac{k_{\mathrm{A}}}{\rho_{\mathrm{A}} c_{A}}
$$

The boundary conditions are,

$$
\begin{aligned}
-k_{\mathrm{A}} \frac{\partial T_{\mathrm{A}}}{\partial x_{s p o t}}+\epsilon_{\mathrm{A}} \sigma T_{\mathrm{A}}^{4} & =\varsigma_{\mathrm{A}} \sigma_{s y s} C_{r} S_{0}\left(\frac{r_{\mathrm{AU}}}{r_{s c}}\right)^{2} \\
T(0, x) & =T_{0} \\
T(t, L) & =T_{0}
\end{aligned}
$$

The initial surface temperature $T_{0}$ is $278 \mathrm{~K}$, which is assumed here to be constant from the surface down to a depth of $L=135 \mathrm{~m}$ inside the asteroid. Fig. 4 shows the sum of the two force components $F_{i r}$ and $F_{\text {light }}$ as a function of the surface temperature for different spot sizes and concentration ratios.

The total deflection acceleration acting on the asteroid is therefore:

$$
\mathbf{u}_{\text {dev }}=\mathbf{u}_{s u b}+\mathbf{u}_{t u g}+\frac{F_{l i g h t}+F_{i r}}{m_{\mathrm{A}}} \hat{\mathbf{v}}_{\mathrm{A}}
$$




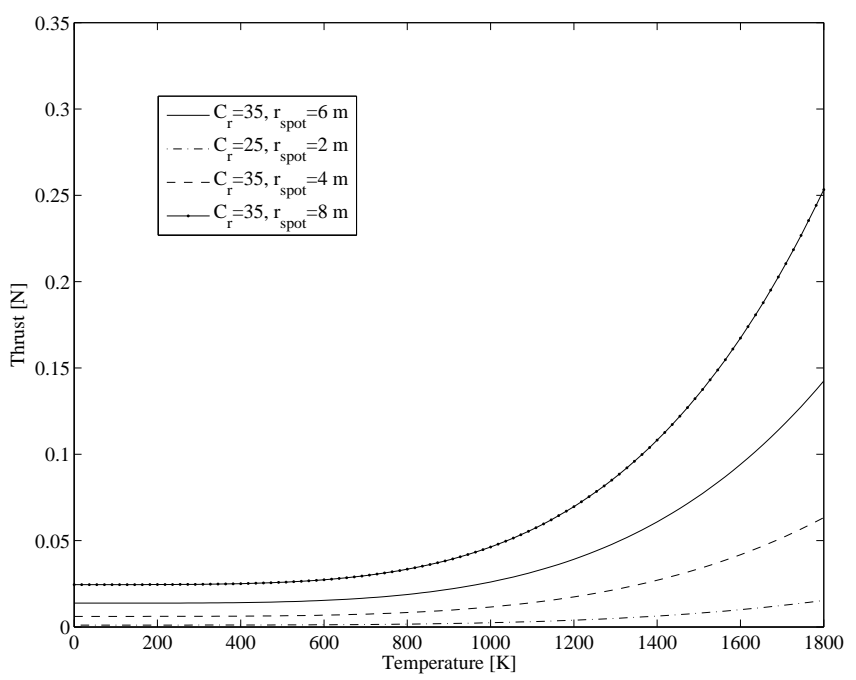

Fig. 4: Total thrust due to combined Yarkovsy and solar pressure effects.

\section{Deflection results}

Fig. 5 shows the differences in required thrust time for a set of warning times between $[4,6] T_{\mathrm{A}}$, or $1294-1941$ days prior to the $t_{\mathrm{MOID}}=64796.56736$ MJD (13 April 2036). The thrust duration is plotted against both the true anomaly of the NEO at the start of the thrust segment (Fig. 5a) and the warning time (Fig. 5b). The two figures confirm a result found by Colombo et al. [7]: the deflection can be maximized by properly timing the beginning of the deviation operations.

Fig. 6 shows the total expelled mass versus a fixed value of the 'system' concentration ratio, i.e. the number of spacecraft times the concentration ratio of each individual spacecraft. It is clearly shown in the plot, that for a larger number of spacecraft (e.g., 10) with a smaller individual concentration ratio (e.g., 50), a greater thrust and hence deflection can be achieved. Fig. 6 also shows that below a given number of spacecraft and a given concentration ratio (lower left corner of the plot), the sublimation is not possible. The power density is too low and the sublimation process does not start.

Figs. $7-8$ show the achieved impact parameter $b$ at a hypothetical MOID with the Earth at $t_{\mathrm{MOID}}=13252.06736$ MJD2000 versus the warning time $\left(t_{\text {warn }}\right)$, for a variable number of spacecraft and a fixed aperture diameter of the primary mirror $\left(d_{\mathrm{M}}\right)$. The system power efficiency $\sigma_{\text {sys }}$ is set to $90 \%$. The thrust leg is assumed to start at $\left(t_{\mathrm{MOID}}-t_{\text {warn }}\right)$ and thrust continuously until $\left(t_{\mathrm{MOID}}-t_{\text {warn }}+t_{\text {thrust }}\right)$. For these tests the orbital parameters of the asteroids have been slightly modified so that the MOID is zero. Following Vasile et al. [39], the impact parameter $b$ is the projection of the deflection on the $b$-plane of the Earth at the time of the MOID. In particular, given the velocity vector of the Earth $\mathbf{v}_{\mathrm{E}}$ and of the relative velocity of the asteroid $\mathbf{U}_{\mathrm{A}}$ we have:

$$
\hat{\eta}_{\mathrm{U}}=\frac{\mathbf{U}_{\mathrm{A}}}{U_{\mathrm{A}}} \quad \hat{\chi}=\frac{\mathbf{v}_{\mathrm{E}} \wedge \hat{\eta}_{\mathrm{U}}}{\left\|\mathbf{v}_{\mathrm{E}} \wedge \hat{\eta}_{\mathrm{U}}\right\|} \quad \hat{\imath}=\hat{\chi} \wedge \hat{\eta}_{\mathrm{U}}
$$

Therefore, the $b$ parameter is defined as $b=\sqrt{\chi^{2}+\iota^{2}}$. 


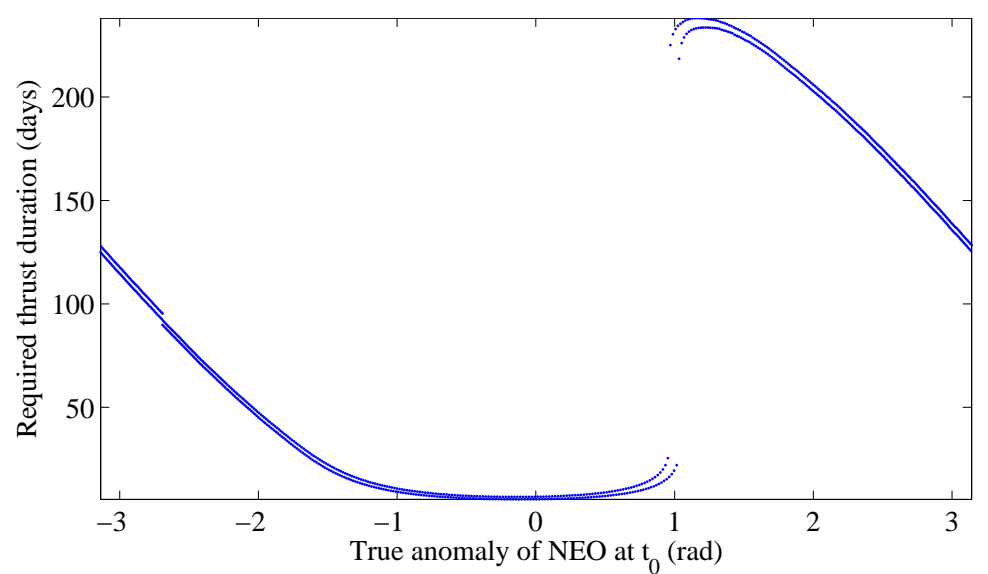

(a)

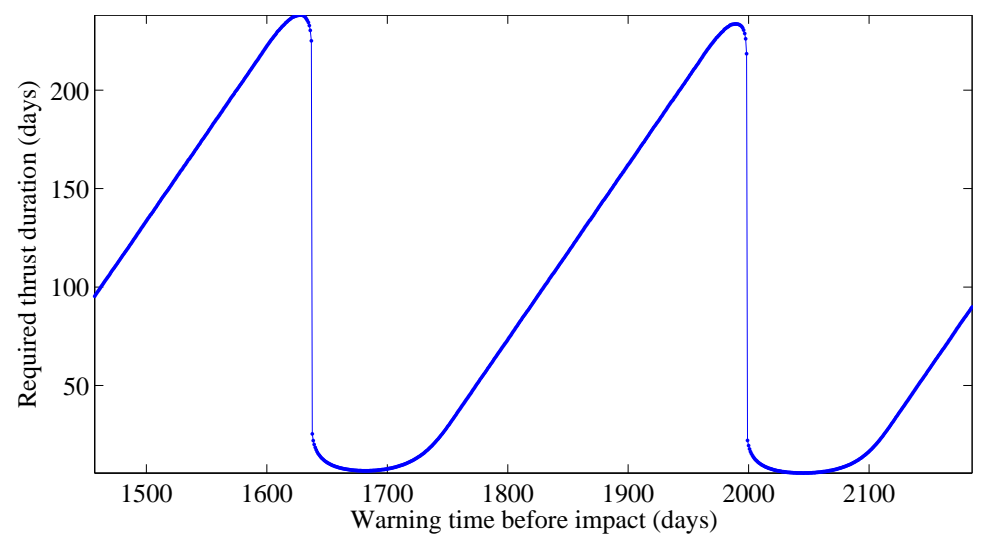

(b)

Fig. 5: Effect of orbital location at the start of thrust period, on the required duration to achieve a deflection of $10000 \mathrm{~km}$ at $t_{\mathrm{MOID}}=64796.56736$ MJD using a single $65 \mathrm{~m}$ mirror with a concentration ratio $C_{r}=98$.

Fig. 7 compares the achievable impact parameter $b$ with and without contamination assuming a variable $C_{r}$. This situation occurs when the mirrors are at a constant distance from the asteroid. In this case the focusing capability of the mirror changes with the distance from the Sun since the angular diameter of the Sun increases, or decreases, as the asteroid moves closer, or farther away. The mirrors are assumed to be at an angular distance of $\varphi=60^{\circ}$ with respect to the $y$ axis and at a distance $\delta r=694.62 \mathrm{~m}$ from the center of the asteroid. Although the mirrors are not directly in the plume, according to Kahle's model the contamination is still quite substantial and the power density quickly falls below the required limit to induce the sublimation. The mirrors will continue to heat up the asteroid but no sublimation will occur.

Fig. 8 compares the achievable impact parameter $b$ with and without contamination assuming a constant $C_{r}$. In this case the mirrors are moving toward the asteroid 


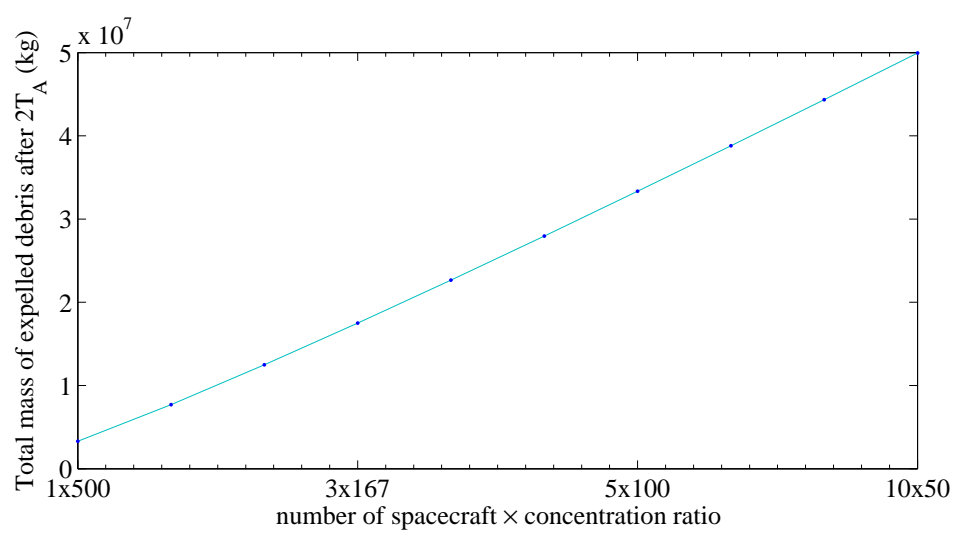

Fig. 6: Total asteroid debris mass expelled by sublimation vs a system concentration factor which is composed of the number of spacecraft in the formation multiplied by the individual concentration ratio of each onboard focusing system. The simulation was run for a $65 \mathrm{~m}$ mirror, $\eta_{s y s}=90 \%$ and with a warning time $=$ thrust time of $2 T_{A}$.

while the asteroid is moving toward the Sun, and vice versa, in order to maintain a constant $C_{r}$. Although this increases the contamination, the performance still improves significantly with respect to the variable $C_{r}$ case. Fig. $8 \mathrm{~b}$ displays a remarkable change in behavior above and below 2 spacecraft. In fact, below 2 spacecraft there is no sublimation at any point along the orbit; the only forces acting on the asteroid are the gravity tug and those induced by solar light (reflection, absorption and Yarkovsky). The same effect can be seen in Fig. $7 \mathrm{~b}$ below the 3 spacecraft line.

Fig. 7a and 8a demonstrate a periodic behavior that is inline with Fig. 5. Due to the contamination, the sublimation ceases very soon, therefore the point at which the deflection action starts can radically alter the performance.

One important thing to note is that increasing the concentration ratio does not improve the deviation. According to the thrust model in Eqs. (7) and (11), the thrust magnitude depends on the input power and surface area illuminated by the beam. As the concentration ratio increases, the area for a fixed mirror size, decreases and therefore the thrust does not improve. On the other hand, superimposing the beams increases the power density and leaves the size of the spot area unchanged. Therefore, rather than increasing the concentration ratio, the ideal strategy would be to increase the number of beams each with a constant concentration ratio.

Fig. 9 shows the impact parameter $b$ that can be achieved with the simple combination of forces given in Eqs. (20), (21) and (18), for a $62 \mathrm{~m}$ aperture diameter mirror. The distance of the mirrors is initially $\delta r=1389.2 \mathrm{~m}$ from the center of the asteroid. Non-homogenous asteroids with materials characterized by a lower sublimation point can represent a problem for this technique but at the same time would lead to a sublimation of the surface at a lower power density. 
Impact parameter $b(\mathrm{~km})$ with contamination, constant $\mathrm{C}_{\mathrm{r}}$ for $d_{M}=62 \mathrm{~m}$

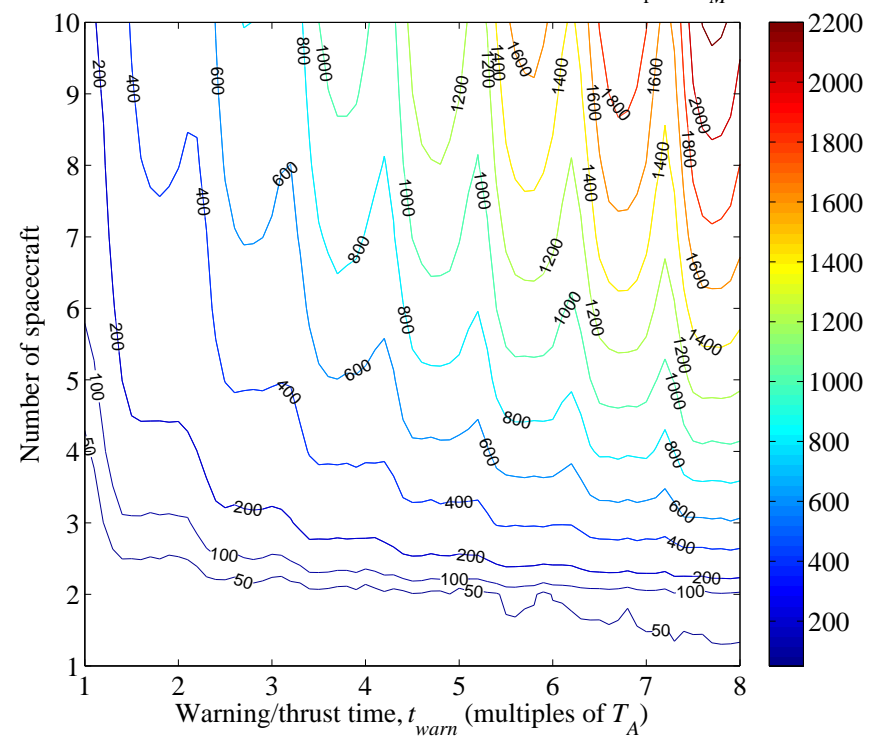

(a) With contamination.

Impact parameter $b(\mathrm{~km})$, no contamination, constant $\mathrm{C}_{\mathrm{r}}$ for $d_{M}=62 \mathrm{~m}$

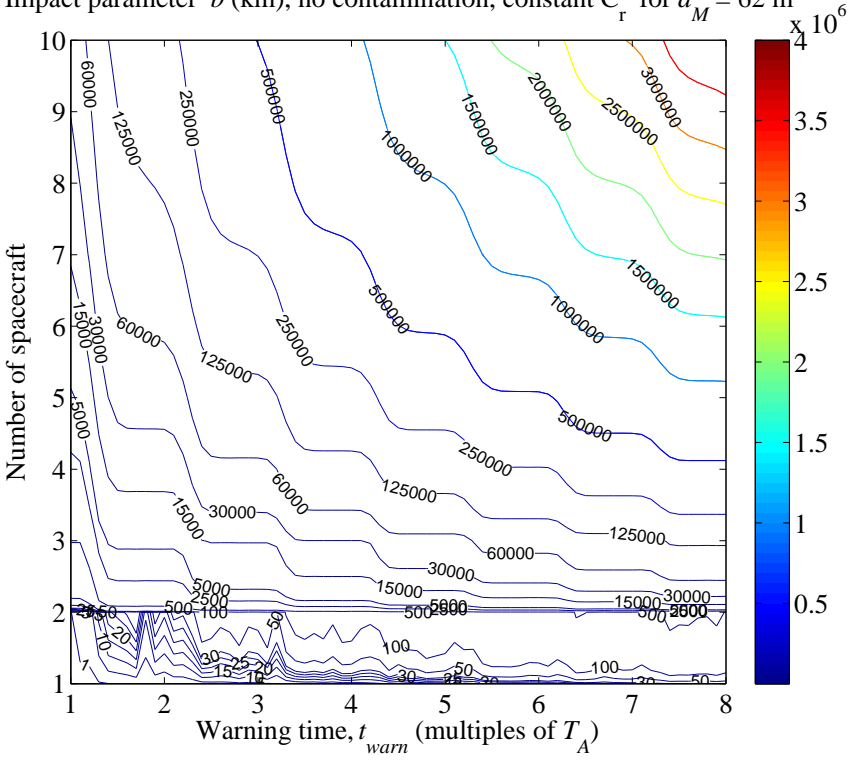

(b) With no contamination.

Fig. 7: Impact parameter for different warning times, variable number of spacecraft and constant $C_{r}$. Aperture of the mirror $d_{\mathrm{M}}=62 \mathrm{~m}$. 
Impact parameter $b(\mathrm{~km})$ with contamination, variable $\mathrm{C}_{\mathrm{r}}$ for $d_{M}=62 \mathrm{~m}$

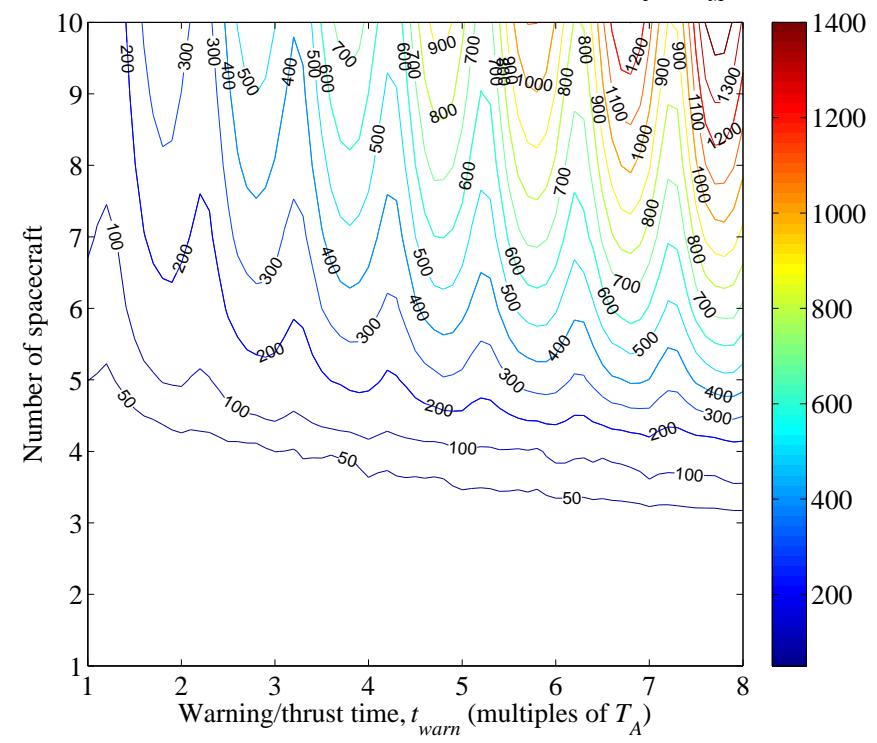

(a) With contamination.

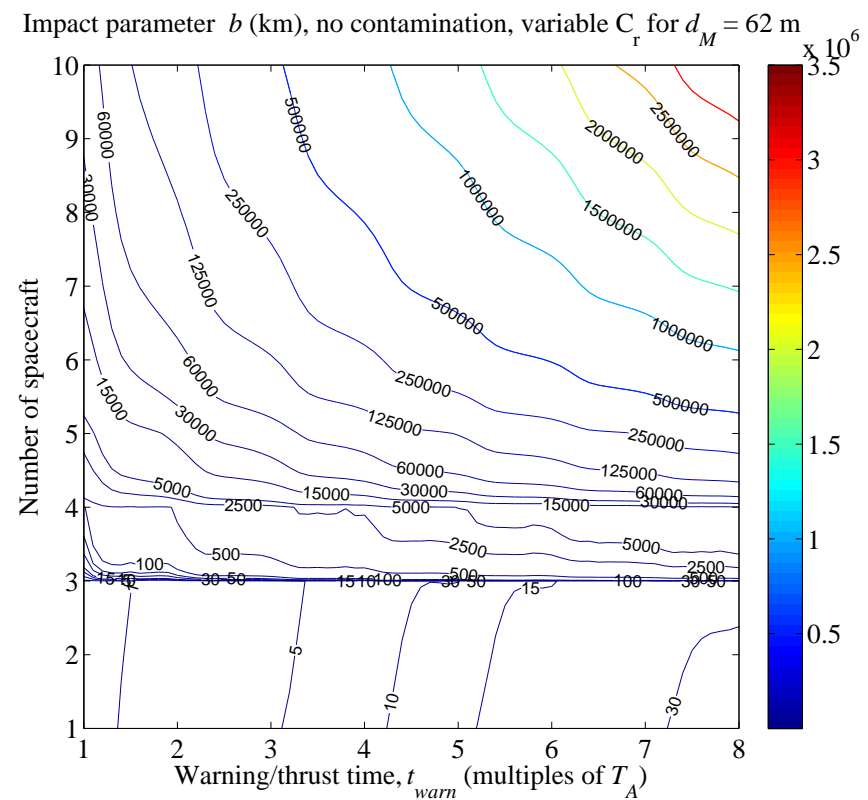

(b) With no contamination.

Fig. 8: Impact parameter for different warning times, variable number of spacecraft and variable $C_{r}$. Aperture of the mirror $d_{\mathrm{M}}=62 \mathrm{~m}$. 
Impact parameter $b(\mathrm{~km})$, only enhanced Yarkovsky, variable $\underset{\mathrm{r}}{\mathrm{C}}$ for $d_{M}=62 \mathrm{~m}$

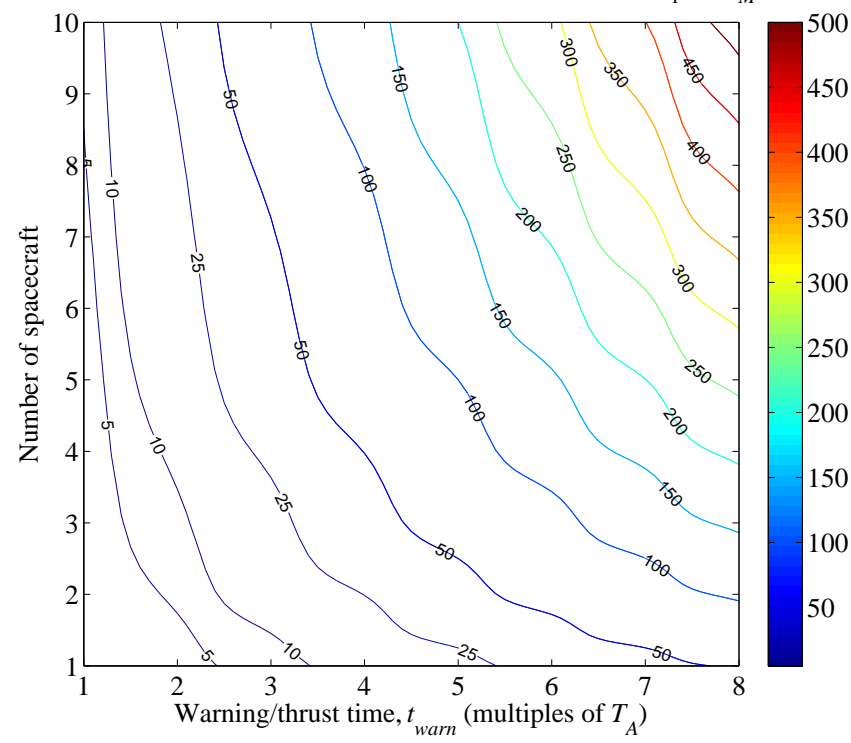

(a) Solar collector with a $62 \mathrm{~m}$ aperture diameter and variable $C_{r}$.

Impact parameter $b(\mathrm{~km})$, only enhanced Yarkovsky, constant $\underset{\mathrm{r}}{\mathrm{r}}$ for $d_{M}=62 \mathrm{~m}$

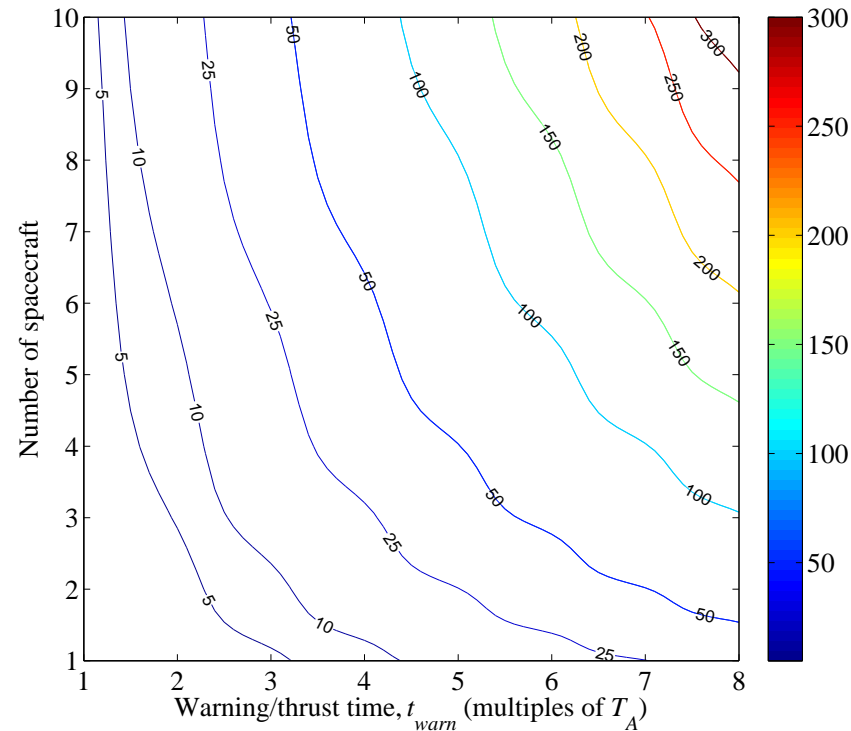

(b) Solar collector with a $62 \mathrm{~m}$ aperture diameter and fixed $C_{r}$

Fig. 9: Enhanced Yarkovsy effect. Impact parameter for different warning times, variable number of spacecraft and variable $C_{r}$. 


\section{Mirror Dynamics and Control}

In order to obtain the desired deviation, the mirrors need to be placed and controlled in the proximity of the asteroid. In particular, the location of the mirror must be such that the plume impingement is minimized and the power density is maximized. In the proximity of the asteroid, in a Hill rotating reference frame, the spacecraft are subject to the force due to solar pressure, the gravity of the asteroid, the gravity of the Sun, the centrifugal and Coriolis forces plus the forces induced by the impingement either with the plume or the re-emitted and reflected light from the asteroid. The mirrors can be designed such that the resultant of all these forces is minimal. An active control can then be added to maintain the spacecraft librating above the surface of the asteroid.

Following the ellipsoidal asteroid model, we assume that the semi-axis $c_{\mathrm{I}}$ is aligned with the $z$-axis of the asteroid Hill frame $\mathcal{A}$ (see Figure 3 ) and that the asteroid rotates around the $z$-axis with angular velocity $w_{\mathrm{A}}$. The gravity field of the asteroid is expressed as the sum of a spherical field plus a second-degree and second-order field [14,31],

$$
U_{20+22}=\frac{\mu_{\mathrm{A}}}{\delta r^{3}}\left(C_{20}\left(1-\frac{3}{2} \cos ^{2} \gamma\right)+3 C_{22} \cos ^{2} \gamma \cos 2 \lambda\right)
$$

where the harmonic coefficients $C_{20}$ and $C_{22}$ are a function of the semi-axes,

$$
\begin{aligned}
& C_{20}=-\frac{1}{10}\left(2 c_{\mathrm{I}}^{2}-a_{\mathrm{I}}^{2}-b_{\mathrm{I}}^{2}\right) \\
& C_{22}=\frac{1}{20}\left(a_{\mathrm{I}}^{2}-b_{\mathrm{I}}^{2}\right)
\end{aligned}
$$

and $\lambda$ is defined as,

$$
\lambda=\arctan \left(\frac{y}{x}\right)+w_{\mathrm{A}} t
$$

with $\gamma=0$ since only the in-plane motion is considered.

If we consider a Hill reference frame $\mathcal{A}$ centered in the barycenter of the asteroid (see Figure 3), the motion of the spacecraft in the proximity of the asteroid is given by:

$$
\begin{aligned}
& \ddot{x}=-\ddot{r}_{A}+2 \dot{\nu} \dot{y}+\dot{\nu}^{2}\left(r_{\mathrm{A}}+x\right)+\ddot{\nu} y-\frac{\mu_{\odot}\left(r_{\mathrm{A}}+x\right)}{r_{s c}^{3}}-\frac{\mu_{\mathrm{A}}}{\delta r^{3}} x+\frac{F_{s_{x}}(x, y, z)}{m_{s c}}+\frac{\partial U_{20+22}}{\partial x} \\
& \ddot{y}=-2 \dot{\nu} \dot{x}-\ddot{\nu}\left(r_{\mathrm{A}}+x\right)+\dot{\nu}^{2} y-\frac{\mu_{\odot}}{r_{s c}^{3}} y-\frac{\mu_{\mathrm{A}}}{\delta r^{3}} y+\frac{F_{s_{y}}(x, y, z)}{m_{s c}}+\frac{\partial U_{20+22}}{\partial y} \\
& \ddot{z}=-\frac{\mu_{\odot}}{r_{s c}^{3}} z-\frac{\mu_{\mathrm{A}}}{\delta r^{3}} z+\frac{F_{s_{z}}(x, y, z)}{m_{s c}}+\frac{\partial U_{20+22}}{\partial z}
\end{aligned}
$$

with,

$$
\begin{aligned}
\ddot{\nu} & =\frac{u_{d e v_{y}}-2 \dot{r}_{\mathrm{A}} r_{\mathrm{A}} \dot{\nu}}{r_{\mathrm{A}}^{2}} \\
\ddot{r}_{\mathrm{A}} & =\dot{\nu}^{2} r_{\mathrm{A}}-\frac{\mu_{\odot}}{r_{\mathrm{A}}^{2}}+u_{d e v_{x}}
\end{aligned}
$$

The force term $\mathbf{F}_{s}=\left[F_{s_{x}} F_{s_{y}} F_{s_{z}}\right]^{\mathrm{T}}$ is made of three contributions: direct light pressure from the Sun $\mathbf{F}_{s r p}$, light pressure from the emitted and reflected light from the asteroid $\mathbf{F}_{\text {light }}$ and the force due to the flow of gas and debris coming from the asteroid $\mathbf{F}_{\text {plume }}$. 
In order to have equilibrium, $\mathbf{F}_{s}$ should be aligned with the gravity vector and apparent forces, but in the opposite direction. Examining Eq. (28c), it is clear that $\ddot{z}$ is zero when $z=0$, therefore in the remainder of this section we will focus on the motion in the $x-y$ plane.

For a direct reflection of the light onto the NEO surface, the focal point is along the mirror-asteroid direction with a magnitude roughly equal to the distance between the mirror and the asteroid. For such a long focal distance, the resulting mirror is almost flat. Assuming a perfect reflection, the force $\mathbf{F}_{\text {srp }}$ is:

$$
\mathbf{F}_{s r p}=2 A_{\mathrm{M}} S_{s r p}\left(\frac{r_{\mathrm{AU}}}{r_{s c}}\right)^{2} \cos ^{2} \beta\left[\begin{array}{c}
\cos \beta \\
\sin \beta \\
0
\end{array}\right]
$$

The angle $\beta$ is the half angle between the asteroid-mirror vector and the Sun-mirror vector (which is approximated by setting it equal to the Sun-asteroid vector).

$$
\beta=\frac{1}{2} \arctan \left(\frac{r_{s / s c, y}}{r_{s / s c, x}}\right)
$$

where $r_{s / s c, y}$ and $r_{s / s c, x}$ are respectively the $y$ and $x$ components of the vector $\mathbf{r}_{s / s c}$.

The force due to the light coming from the asteroid can be expressed in the following form:

$\mathbf{F}_{\text {light }}=2 n_{\text {spot }} \frac{\left(\Lambda \frac{\epsilon_{\mathrm{A}} \sigma T_{\mathrm{A}}^{4}}{c}+\Lambda_{\text {light }}\left(1-\varsigma_{\mathrm{A}}\right) \sigma_{s y s} C_{r} S_{s r p}\left(\frac{r_{\mathrm{AU}}}{r_{s c}}\right)^{2}\right) A_{s p o t}}{2 \pi r_{s / s c}^{2}} A_{\mathrm{M}} \cos ^{2} \beta\left[\begin{array}{c}\cos \beta \\ \sin \beta \\ 0\end{array}\right]$

where $n_{\text {spot }}$ is the number of spot areas emitting and reflecting light. The assumption is made that the radiation is uniformly distributed over a hemisphere. Note that in the case of the enhanced Yarkovsky effect $n_{\text {spot }}=n_{s c}$ while in the case of sublimation $n_{\text {spot }}=1$. The force due to the two contributions of light pressure is aligned with the asteroid-mirror direction. Moreover, the modulus of the light pressure is a function of the distance from the Sun.

If the flow rate per unit area at distance $r_{s / s c}$ is $\left(2 \rho_{\exp }\left(r_{s / s c}, \varphi\right) \bar{v}\right)$ and all the particles stick to the surface of the mirror then the force $\mathbf{F}_{\text {plume }}$ is:

$$
\mathbf{F}_{\text {plume }}=4 \rho_{\exp }\left(r_{s / s c}, \varphi\right) \bar{v}^{2} A_{\mathrm{M}} \cos \psi_{v f} \hat{\mathbf{r}}_{s / s c}
$$

and is aligned with the $\mathbf{r}_{s / s c}$. However, the flow rate depends on the power density and therefore on the distance from the Sun. Given these equations, the resultant of all the forces acting on the spacecraft is not zero and in particular the difference between gravity and $\mathbf{F}_{s}$ is a function of time. Therefore, an active control is required to maintain the position of the spacecraft with respect to the asteroid.

The force due to the light coming from the asteroid can be up to $1.6948 \times 10^{-5} \mathrm{~N}$ at perihelion for a $C_{r}=35, r_{s / s c}=857 \mathrm{~m}$ and $n_{s c}=1$ and grows up to $2.0337 \times 10^{-4} \mathrm{~N}$ for $n_{s c}=12$. It is therefore two to three orders of magnitude lower than the force in Eq. (31), which, for similar operating conditions, is $2.636 \times 10^{-2} \mathrm{~N}$. The force due to gas and debris instead is $F_{\text {plume }}=3.533 \times 10^{-3} \mathrm{~N}$ for $n_{s c}=4$ and $F_{\text {plume }}=3.727 \times 10^{-2} \mathrm{~N}$ for $n_{s c}=9$, with $C_{r}=100$, the mirrors at a distance $r_{s / s c}=507 \mathrm{~m}$ and an elevation angle of $60^{\circ}$. Therefore, the force due to the plume is not negligible for a high number of spacecraft. 
If we assume that $F_{\text {light }}$, centrifugal and Coriolis forces are negligible compared to solar pressure, gravity of the asteroid, and plume and that any non-spherical terms in the gravity field expansion result in only a small perturbation, then we can build a simple control law based on the Lyapunov control function:

$$
V=\frac{1}{2} \delta v^{2}+\frac{1}{2} K\left(\left(x-x_{\mathrm{AEP}}\right)^{2}+\left(y-y_{\mathrm{AEP}}\right)^{2}+\left(z-z_{\mathrm{AEP}}\right)^{2}\right)
$$

where $\delta \mathbf{r}_{\mathrm{AEP}}=\left[x_{\mathrm{AEP}}, y_{\mathrm{AEP}}, z_{\mathrm{AEP}}\right]^{\mathrm{T}}$ are the coordinates of the artificial equilibrium point at which we want to place the mirror (in the Hill frame). Now if there exist a control $\mathbf{u}$ such that $d V / d t<0$ then we can maintain the mirror in the proximity of the artificial equilibrium point. A possible control is given by:

$$
\mathbf{u}=-\left(-\frac{\mu_{\mathrm{A}}}{\delta r^{3}} \delta \mathbf{r}+\frac{\mathbf{F}_{s r p}}{m_{s c}}+\frac{\mathbf{F}_{p l u m e}}{m_{s c}}\right)-K\left(\delta \mathbf{r}-\delta \mathbf{r}_{\mathrm{AEP}}\right)-c_{d} \delta \mathbf{v}
$$

The total derivative of the function $V$ is:

$$
\begin{aligned}
& \frac{d V}{d t}=\delta \mathbf{v}^{T} \delta \dot{\mathbf{v}}+k\left(\delta \mathbf{r}-\delta \mathbf{r}_{\mathrm{AEP}}\right)^{T} \delta \mathbf{v} \\
&=\delta \mathbf{v}^{T}(-\frac{\mu_{\mathrm{A}}}{\delta r^{3}} \delta \mathbf{r}+\frac{\mathbf{F}_{s r p}}{m_{s c}}+\frac{\mathbf{F}_{\text {plume }}}{m_{s c}}-\left(-\frac{\mu_{\mathrm{A}}}{\delta r^{3}} \delta \mathbf{r}+\frac{\mathbf{F}_{s r p}}{m_{s c}}+\frac{\mathbf{F}_{p l u m e}}{m_{s c}}\right) \\
&\left.\quad-K\left(\delta \mathbf{r}-\delta \mathbf{r}_{\mathrm{AEP}}\right)-c_{d} \delta \mathbf{v}\right)+K\left(\delta \mathbf{r}-\delta \mathbf{r}_{\mathrm{AEP}}\right)^{T} \delta \mathbf{v} \\
&=-c_{d} \delta \mathbf{v}^{T} \delta \mathbf{v}<0
\end{aligned}
$$

where $\delta \mathbf{v}=[\dot{x}, \dot{y}, \dot{z}]^{\mathrm{T}}$ is the relative velocity of the spacecraft in the asteroid Hill reference frame $\mathcal{A}$.

We can now introduce the control equation (36) into the full dynamic model in Eq. (28) and test the validity of the assumption on that the perturbations given by centrifugal, Coriolis forces, light coming from the asteroid and aspherical gravity field are indeed small. The result is represented in Figs. 10a, 11a for the case in which the AEP is maintained at a fixed distance from the asteroid. Figs. 10b, 11b represent, instead, the result of the application of the Lyapunov controller when a different AEP is selected along the the asteroid-mirror direction at different instants of time during the year.

The elastic coefficient $K$ for both cases was chosen to be $10^{-6}$ while the dissipative coefficient $c_{d}$ was set to $10^{-5}$. The latter strategy maintains a constant concentration ratio while the asteroid moves closer and farther from the Sun. In order to maintain a constant $C_{r}$, the focal length has to be modified according to the angular diameter of the Sun seen from the mirror.

Fig. 10b shows that the the controller is able to maintain the mirror in close proximity to the radial direction, effectively chasing the position of the AEP. Figs. 11b and 12 represent the modulus of the thrust and the mass consumption for a one year of operation of a $3005 \mathrm{~kg}$ spacecraft, carrying a mirror with an aperture diameter of $62 \mathrm{~m}$. The required peak thrust is above $40 \mathrm{mN}$, with a total mass consumption of about $15.0 \mathrm{~kg}$, assuming an engine $I_{s p}$ of $4500 \mathrm{~s}$, for maintaining a fixed position. For chasing the position of the AEP's, instead, the required peak thrust is about $35 \mathrm{mN}$, with a total mass consumption of about $12.0 \mathrm{~kg}$ a year. 
These figures demonstrate that with a very small electric propulsion system, the mirror position can be maintained at the desired proximity to the asteroid. Note that the thrust is acting towards the asteroid mainly to compensate for the force due to solar pressure.

\section{Final Remarks}

This paper presented an analysis of the performance of an asteroid deflection method based on the sublimation of surface matter through a concentrated solar beam. According to the available model for the sublimation process, the obtainable deflection is limited by the contamination of the reflector even if the reflector is not directly in the plume of expelled gas. Using multiple spacecraft improves the deflection but does not solve the contamination problem. On the other hand, this study showed that even when the power density is not enough to ignite the sublimation process, the increase in the Yarkovsky effect combined with the enhanced light pressure generates a reasonable deflection comparable to the solution generated with the sublimation of the surface in the case of contamination of the mirrors. This study also demonstrated that a constant concentration ratio solution, with variable distance of the mirrors from the target, is more effective than a constant position solution. A Lyapunov controller was developed to allow the control of the position of the mirrors at a very low propellant cost.

The results in this paper demonstrate that the solar sublimation system is severely penalized by the contamination of the reflector. Although, the system is always able to generate a deflection of several hundred kilometers, the total mass into space can make the system non-competitive against other deflection methods. A more accurate contamination model is necessary to assess the actual applicability of this deflection approach. A solution to the contamination problem would make this deflection approach extremely effective for medium size asteroids. On the other hand, a contained system with a small number of spacecraft enhancing the Yarkovsky effect can provide a deflection comparable to a gravity tractor for the same class of asteroids.

Acknowledgements The authors would like to thank Dr Leopold Summerer from the ESA/ESTEC Advanced Concepts Team for his support and advice.

\section{References}

1. Barucci, M., Yoshikawa, M., Michel, P., Kawagushi, J., Yano, H., Brucato, J., Franchi, I., Dotto, E., Fulchignoni, M., Ulamec, S.: MARCO POLO: Near Earth Object sample return mission. Experimental Astronomy 23(3), 785-808 (2009)

2. Battin, R.H.: An Introduction to the Mathematics and Methods of Astrodynamics. Revised edn. AIAA Education Series (1999)

3. Brož, M.: Yarkovsky effect and the dynamics of the solar system. PhD thesis, Faculty of Mathematics and Physics, Astronomical Institute, Charles University, Prague, Czech Republic (2006)

4. Carusi, A., Valsecchi, G.B., D’abramo, G., Bottini, A.: Deflecting NEOs in route of collision with the Earth. Icarus 159(2), 417-422 (2002). DOI 10.1006/icar.2002.6906

5. Chesley, S.R.: Potential impact detection for near-Earth asteroids: The case of 99942 Apophis (2004 $\mathrm{MN}_{4}$ ). In: Asteroids, Comets, Meteors Proceedings, vol. 229, pp. 215-228. IAU Symposium (2005)

6. Colombo, C., Vasile, M., Radice, G.: Optimal low-thrust trajectories to asteroids through an algorithm based on differential dynamic programming. Celestial Mechanics and Dynamical Astronomy 105(1-3), 75-112 (2009) 


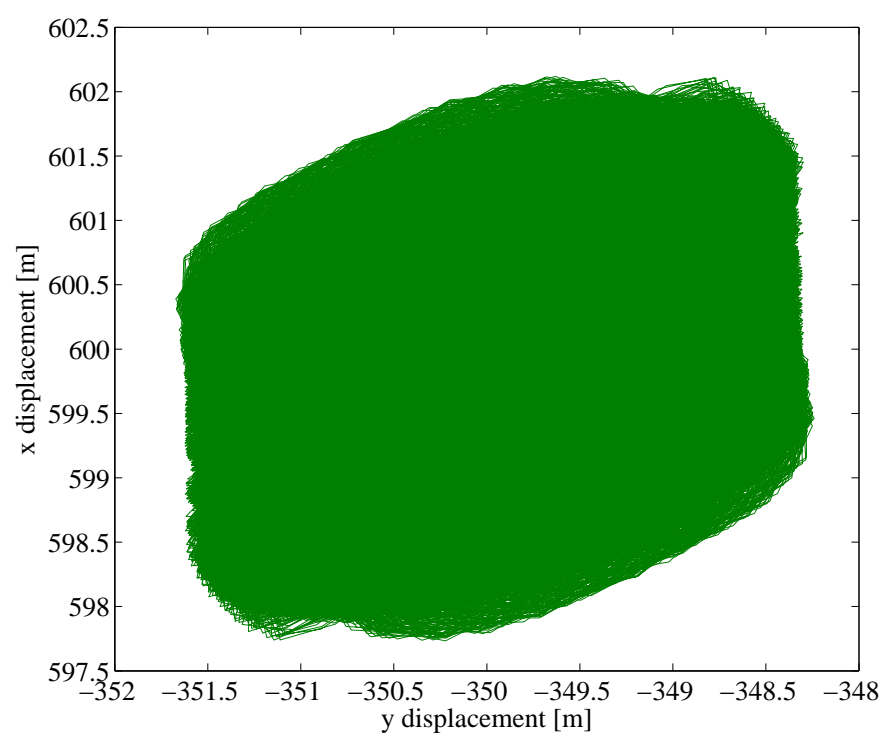

(a) Fixed position.

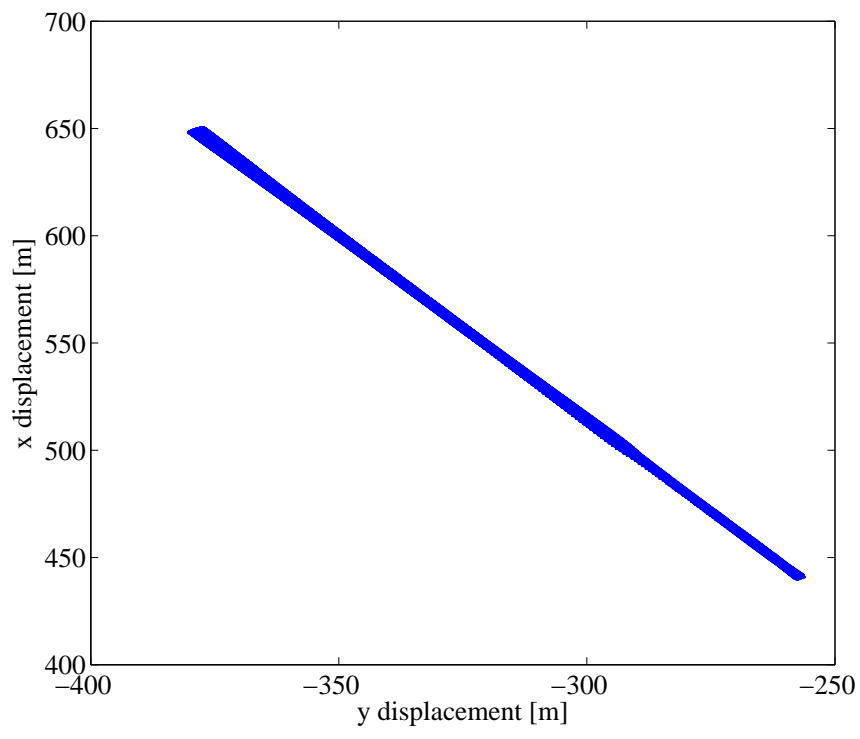

(b) Variable position.

Fig. 10: Variation in position over one orbit shown in the local mirror reference frame, for Lyapunov controlled AEPs: a) fixed AEP maintenance, b) AEP position tracking. 


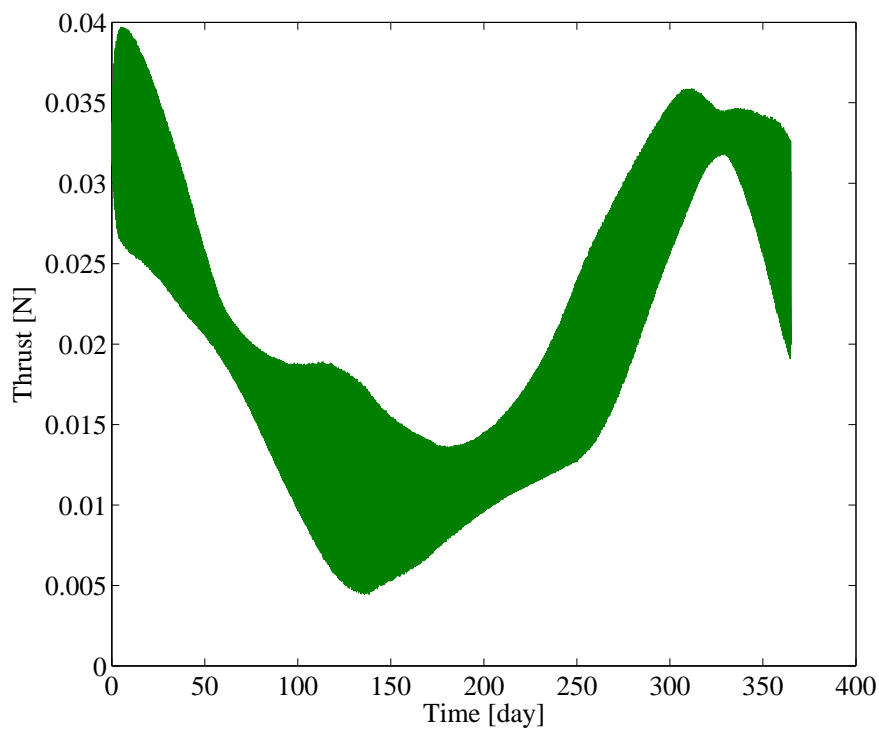

(a) Fixed position.

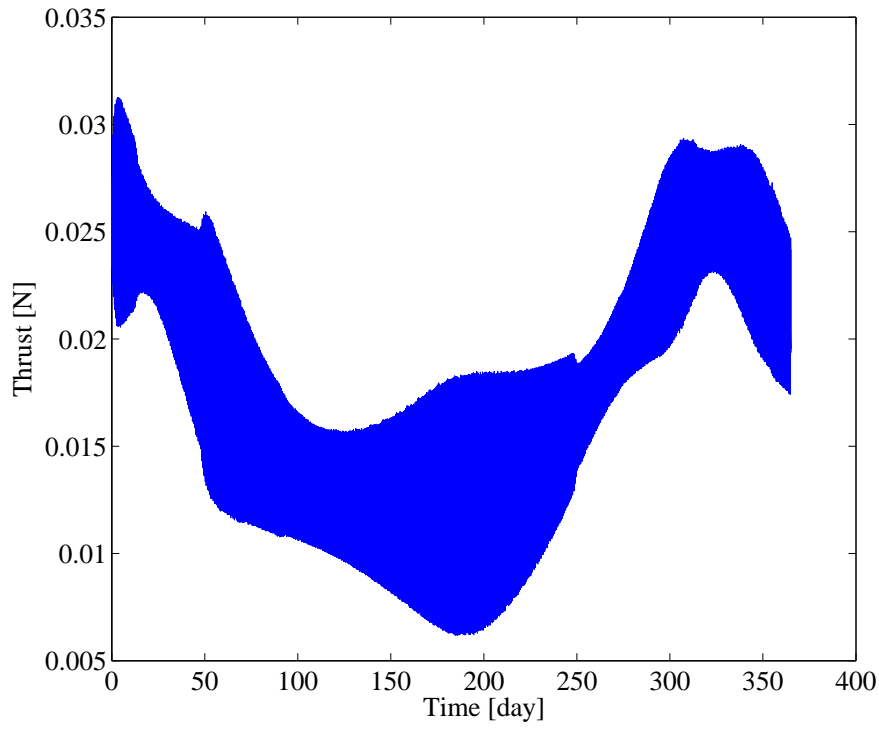

(b) Variable position.

Fig. 11: Thrust magnitude for Lyapunov controlled AEPs: a) fixed AEP maintenance, b) AEP position tracking 


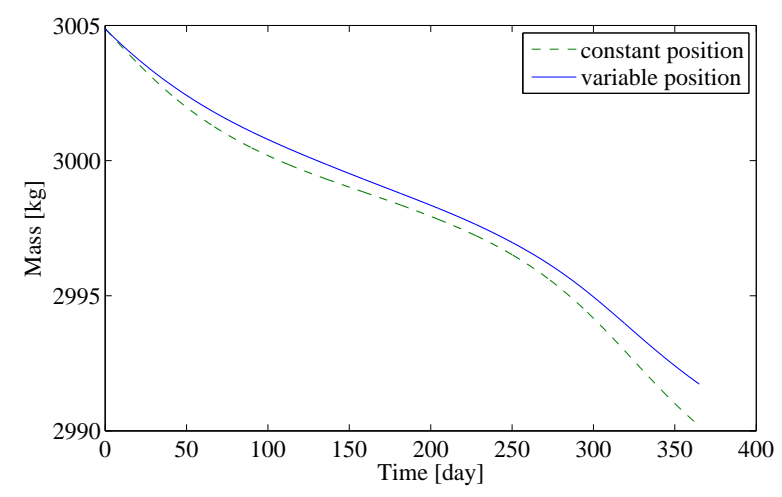

Fig. 12: Mass consumption for Lyapunov controlled AEPs.

7. Colombo, C., Vasile, M., Radice, G.: Semi-analytical solution for the optimal low-thrust deflection of Near-Earth Objects. Journal of Guidance, Control and Dynamics 32(3), 796-809 (2009). DOI 10.2514/1.40363

8. Conway, B.: Near-optimal deflection of Earth-approaching asteroids. Journal of Guidance, Control and Dynamics 24(5), 1035-1037 (2001)

9. Delbò, M., Cellino, A., Tedesco, E.: Albedo and size determination of potentially hazardous asteroids: (99942) Apophis. Icarus 188, 266-269 (2007). DOI 10.1016/j.icarus.2006.12.024

10. Giorgini, J., Benner, L., Ostroa, S., Nolan, M., Busch, M.: Predicting the earth encounters of (99942) apophis. Icarus 193, 1-19 (2008). DOI 10.1016/j.icarus.2007.09.012

11. Glassmeier, K.H., Boehnhardt, H., Koschny, D., Kührt, E., Richter, I.: The Rosetta mission: Flying towards the origin of the solar system. Space Science Reviews 128(1-4), 1-21 (2007)

12. Hall, C.D., Ross, I.M.: Dynamics and control problems in the deflection of Near-Earth Objects. Advances in the Astronautical Sciences 67(640), 1-18 (1997)

13. Hampton, D., Baer, J., Huisjen, M., Varner, C., Delamere, A., Wellnitz, D., A'Hearn, M., Klaasen, K.: An overview of the instrument suite for the deep impact mission. Space Science Reviews 117(1-2), 43-93 (2005)

14. Hu, W., Scheeres, D.J.: Spacecraft motion about slowly rotating asteroids. Journal of Guidance, Control and Dynamics 25(4), 765-775 (2002)

15. IAU Minor Planet Center: Observer services: Neos. http://www.cfa.harvard.edu/iau/mpc.html (2010)

16. Izzo, D.: On the deflection of potentially hazardous objects. In: AIAA/AAS Space Flight Mechanics Conference. Copper Mountain, Colorado (2005)

17. Kahle, R., Kührt, E., Hahn, G., Knollenberg, J.: Physical limits of solar collectors in deflecting Earth-threatening asteroids. Aerospace Science and Technology 10, 253-263 (2006). DOI 10.1016/j.ast.2005.12.004

18. Legge, H., Boettcher, R.: Modelling control thrust plume flow and impingement. In: International Symposium on Rarefied Gas Dynamics, pp. 983-992 (1982)

19. Lunan, D.: Need we protect Earth from space objects and if so, how? Space Policy 8(1), 90-91 (1992)

20. Maddock, C., Vasile, M.: Design of optimal spacecraft-asteorid formations through a hybrid global optimization approach. Journal of Intelligent Computing and Cybernetics 1(2), 239-268 (2008). DOI 10.1108/17563780810874735

21. McAdams, J.V., Dunham, D.W., Mosher, L.E., Ray, J.C., Antreasian, P.G., Helfrich, C.E., Miller, J.K.: Maneuver history for the NEAR mission - Launch through Eros orbit insertion. In: Proceedings of the AIAA/AAS Astrodynamics Specialist Conference, AIAA2000-4141 (2000)

22. Melosh, H.J., Nemchinov, I.V.: Solar asteroid diversion. Nature 366, 21-22 (1993)

23. Melosh, H.J., Nemchinov, I.V., Zetzer, Y.I.: Non-nuclear strategies for deflecting comets and asteroids. In: Gehrels, T. (ed.) Hazard due to comets and asteroids, pp. 1111-1132. University of Arizona Press (1994) 
24. Nakamura, A.M., Michel, P.: Asteroids and their collisional disruption. In: Lecture Notes in Physics, Small Bodies in Planetary Systems, pp. 1-27. Springer Berlin / Heidelberg (2009)

25. NASA Near Earth Object program: 99942 Apophis (2004 MN4) impact risk. Online database, http://neo.jpl.nasa.gov/risk/a99942.html (2010)

26. Near-Earth Object Science Definition Team: Study to determine the feasibility of extending the search for Near-Earth Objects to smaller limiting diameters. Tech. rep., National Aeronautics and Space Administration (NASA) (2003)

27. Park, S.Y., Mazanek, D.D.: Deflection of Earth-crossing asteroids/comets using rendezvous spacecraft and laser ablation. Journal of Astronautical Sciences 53(1), 21-37 (2005)

28. Perozzi, E., Casalino, L., Colasurdo, G., Rossi, A., Valsecchi, G.: Resonant fly-by missions to Near Earth Asteroids. Celestial Mechanics and Dynamical Astronomy 83(1-4) (2002)

29. Rayman, M., Varghese, P., Lehman, D., Livesay, L.: Results from the Deep Space 1 technology validation mission. Acta Astronautica 47(2), 475-487 (2000)

30. Remo, J.L.: Classifying and modeling NEO material properties and interactions. In: Gehrels, T., Matthews, M.S., Schumann, A. (eds.) Hazards due to comets and asteroids, Space Science Series, pp. 551-596. University of Arizona Press, Tucson, AZ (1994)

31. Rossi, A., Marzari, F., Farinella, P.: Orbital evolution around irregular bodies. Earth, Planets, Space 51, 1173-1180 (1999)

32. Russell, C.T., Capaccioni, F., Coradini, A., de Sanctis, M.C., Feldman, W.C., Jaumann, R., Keller, H.U., McCord, T.B., McFadden, L.A., Mottola, S., Pieters, C.M., Prettyman, T.H., Raymond, C.A., Sykes, M.V., Smith, D.E., Zuber, M.T.: Dawn mission to Vesta and Ceres. Earth, Moon, and Planets 101(1-2), 65-91 (2007)

33. Sanchez, J.P., Vasile, M., Radice, G.: Consequences of asteroid fragmentation during impact hazard mitigation. Journal of Guidance, Control and Dynamics (2010). DOI $10.2514 / 1.43868$

34. Sanchez Cuartielles, J.P., Colombo, C., Vasile, M., Radice, G.: Multi-criteria comparison among several mitigation strategies for dangerous Near Earth Objects. Journal of Guidance, Control and Dynamics 32(1), 121-142 (2009). DOI 10.2514/1.36774

35. Scheeres, D.J., Schweickart, R.L.: The mechanics of moving asteroids. In: Planetary Defense Conference: Protecting Earth from Asteroids. AIAA, Orange County, California (2004)

36. Schweickart, R.L.: A call to (considered) action: International space development conference. Occasional Paper 0501, B612 Foundation (2005)

37. Shengping Gong, J.L., BaoYin, H.: Formation flying solar-sail gravity tractors in displaced orbit for towing near-earth asteroids. Celestial Mechanics and Dynamical Astronomy 105(1-3), 159-177 (2009)

38. Stephan, T.: Assessing the elemental composition of comet $81 \mathrm{P} / \mathrm{Wild} 2$ by analyzing dust collected by Stardust. Space Science Reviews 138(1-4), 247-258 (2008)

39. Vasile, M., Colombo, C.: Optimal impact strategies for asteroid deflection. Journal of Guidance, Control and Dynamics 31(4), 858-872 (2008). DOI 10.2514/1.33432

40. Vasile, M., Maddock, C., Radice, G., McInnes, C.: Call for ideas: NEO Encounter 2029, NEO deflection through a multi-mirror system. Tech. Rep. Ariadna ID: 08/4301, Contract Number: 21665/08/NL/CB, ESA/ESTEC Advanced Concepts Team (2009)

41. Vokrouhlicky, D., Chesley, S., Milani, A.: On the observability of radiation forces acting on near-Earth asteroids. Celestial Mechanics and Dynamical Astronomy 81(1-2), 149-165 (2001)

42. Wang, J., Davis, A., Clayton, R., Hashimoto, A.: Evaporation of single crystal forsterite: Evaporation kinetics, magnesium isotope fractionation, and implications of mass-dependent isotopic fractionation of a diffusion-controlled reservoir. Geochimica et Cosmochimica Acta 63(6), 953-966 (1999). DOI 10.1016/S0016-7037(98)00286-5

43. Yoo, S.M., Song, Y.J., Park, S.Y., Choi, K.H.: Spacecraft formation flying for earthcrossing object deflections using a power limited laser ablating. Advances in Space Research 43, 1873-1889 (2009). DOI 10.1016/j.asr.2009.03.025 